\title{
Design of Tower Management Software Based on Java language
}

\author{
Yingqi Zhao \\ Sontian College of Guangzhou University, Zengcheng Guangzhou, 511370, China
}

\begin{abstract}
Key words: Computer management system, SpringMVC infrastructure, Distributed file server,
\end{abstract} Java Language.

\begin{abstract}
The wisdom of public security is an important part of the wisdom of the city, to mobilize the masses to patrol and control is the key of public security. In order to solve the difficult issue of people-management, need to promote the management technology of information by using the methods of science and technology, Internet. The tower-management system arises at the historic moment. The system is equipped with intelligent device, GIS information system, regional grid system, supporting the use of GPS positioning system and service monitoring command center, it can manage and dispatch all the people of the region for daily-scheduling. The kernel development kits of the system is based on Java language, combined with J2EE technology architecture, and mobile communication technology, database sharding technique, NoSQL database technology, massive distributed file service technology. The tower management system includes web service terminal and mobile client app in two parts. The experimental results show that the system has the characteristics of safety, reliability, intelligence expansion, standard open, easy to use and so on.
\end{abstract}

\section{Introduction}

"Group anti-group management tower management system software" system is established based on the actual needs of Shenzhen Longhua branch, website: www.qunfangtong.org, designed to allow police officers and people through the "Internet + police" shortcut mode, enjoy Public security public service speed and change, improve the efficiency of public security police handling, to achieve the wisdom of the fingertips police. The project is mainly aimed at the police business with the closest relationship with the police. There are eight items on the line: organization management, personnel management, regional management, scheduling management, on-the-job monitoring, service command, vehicle acquisition and integral assessment. After the system is on the line, it is expected to use more than 100,000 people.

Group defense system includes WEB background management and APP mobile client in two parts, in the system architecture belongs to the application support layer, the specific realization of the main functions include:

System management: institutional management, personnel management, regional management, scheduling management, service management, on-the-job monitoring, service command, integral assessment.

APP side: get off work punch, attendance management, task reception, service reporting, a key alarm, service circle.

The system completion function mainly includes the construction of the organization and the organization management system, the personnel information system construction, the regional planning management system construction, the vehicle information collection system, the information reporting, the circulation, the process design, and the large data analysis system for the personnel, the area and the time. The real realization of the fingertips on the wisdom of the police design goals is achieved. 


\section{System overall design structure}

\section{The overall architecture of the system is divided into logical}

Group management group management system uses hierarchical classification, service approach to build [2-4]. The system supports a browser-mode management access and intelligent terminal APP access.

The overall architecture of the system adopts hierarchical design idea. At the level, the system includes: access layer, application layer, application support layer, data layer, infrastructure layer (including system software support layer, hardware and network layer). The system also includes three support systems: information security system, operational maintenance system, standard system and external system integration and access.

Among them, the access portal layer, including the Internet WEB access and smart phones, tablet PCs and other mobile access, you can log on through the account password, with authentication and other security measures.

The application layer is a specific functional module, including the tower organization and management module, patrol area grid module, intelligent scheduling and attendance management module, service command management module, integral management module and reporting subsystem.

Application support layer in the overall structure of the bearing bear the key role in the application layer and the system between the support layers. Application support layer provide some public functions to facilitate the realization of the application function, divided into management and APP fingertips function side.

Data layer contains the system database, service database and distributed picture library, storage of public security bureau, the police station a lot of data information.

\section{Overall network architecture}

The tower system network architecture includes the data transmission network and the application server network in the content. The data transmission network is based on the communication carrier network (including wireless data transmission and fixed network data transmission), and the wireless data transmission needs to provide the relevant data package.

The application server network primarily isolates external network attacks and implements external service requests, as well as internal business module interactions.

The overall network architecture is hierarchical, the first layer for the Public Security Bureau room server layer, with access server, application server, database and image server, they switch and routing, and set the firewall to protect the internal network, and finally by fiber access The Internet, the realization of data collection and transmission; the second floor for the Public Security Bureau command center, with command electronic screen, monitoring center and system management machine, command staff can monitor the scene according to the situation and staff distribution, So that the task to complete efficiently; the third floor for the squadron police room layer, in the region of the security squadron are equipped with monitoring command center, in the jurisdiction of the area to achieve electronic monitoring and intelligent scheduling; the fourth layer is the wireless access layer, Each of the police officers carrying out patrols and the masses on the smart phone to download "group defense" software, encountered all kinds of emergencies (such as fire or criminal cases, etc.), to collect clues to the case and found some major security risks and so can The first time reported to the Public Security Bureau command center, to achieve the purpose of mass defense.

\section{The overall application deployment architecture}

The system adopts BS, APP + Server mixed mode development, mainly related to APP deployment and server software deployment. 
The browser mode is mainly provided to the system administrator to use the maintenance system, as well as the group defense group tower monitoring and other scene demands, the use of personnel through the browser (IE8 above, Chrome) access system.

APP support Android smart phone, the user through the download site and the major application market download and install APP.

The server software includes a four-tier architecture, the forefront of the agent and load balancing system, the use of Nginx load balancing software, deployed in the general-purpose computing server.

The second layer is WEB and APP access system, this part carries all users access authentication, authentication and other basic, the use of distributed cluster deployment, to provide massive user requests to access, while the cluster to enhance system reliability, Point failure, as shown in Figure 1. Database cluster, use NoSQL clustering technology and file server clustering technology.

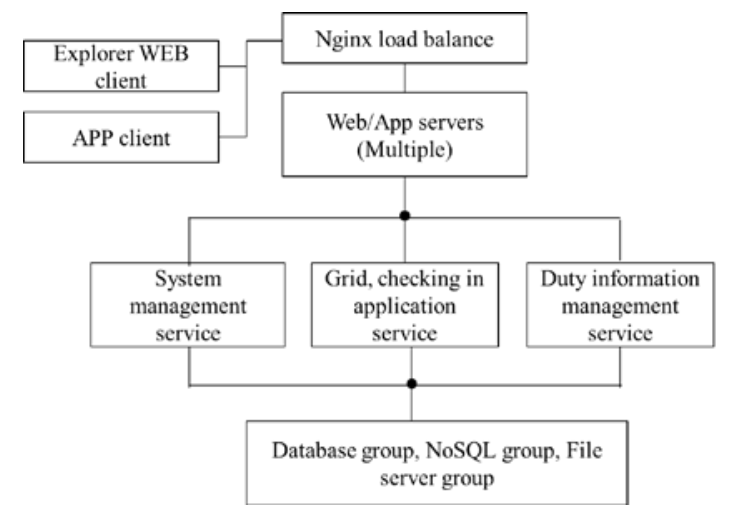

Fig. 1. Nginx Load balance system

The third layer is the application server cluster divided according to the service type. One application server adopts the single point deployment, and the subsequent deployment can be distributed according to the actual business needs of the branch office.

The fourth layer is the data layer, which mainly includes Mysql database cluster, NoSQL cluster (Cassandra, HBase) and distributed file system deployment. Three systems are used on three servers.

\section{General information resource structure}

The information resources of the tower management system include: user basic information, user history information (login, attendance, and track), service information (task, service, and feedback), and picture information, as shown in Table 1.

Table 1. System Information Resource Architecture

\begin{tabular}{|c|c|c|}
\hline \multirow{2}{*}{ Format } & Category & Scope \\
\hline \multirow{2}{*}{ Structured } & Data table(relation) & User basic information, real-time data \\
\cline { 2 - 3 } & Data table (NoSQL) & Historical information \\
\cline { 2 - 3 } & Structured log & Running status, audit log, operation information \\
\hline Non-structured & Pictures & Duty pictures \\
\cline { 2 - 3 } & Videos & Duty videos \\
\hline
\end{tabular}

\section{Tower system organization and personnel management}

\section{Tower organization management model}


The management objectives of the group management are to participate in the various levels of the group, including the branch leaders, the security brigade, squadrons and unit personnel, as well as patrolmen and joint guards. The system is designed to better serve and manage every member of this group.

The tower organization staff management subsystem mainly includes the following functions, as shown in Figure 2:

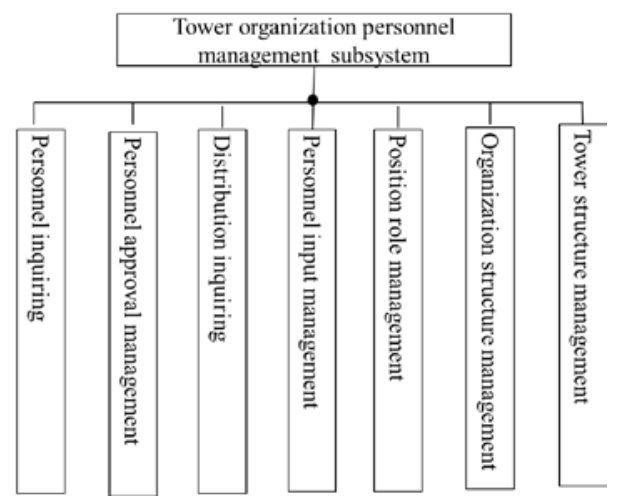

Fig. 2. Tower organization personnel management subsystem

\section{Tower organization management model}

Tower structure is the basic management model of organization and personnel in tower management. It is a top-down management model, which shows the organization level in the form of pyramid hierarchy. One level represents a kind of organization. , The organization block through different colors to show. Each layer of the tower level shows the organization name and job list.

System management using tower hierarchical model, top-down respectively for the detachment, brigade, squadron and unit, each level set different operating authority, to achieve the jurisdiction of the regional group of anti-group rule forces unified management. System support for new, modify, adjust, delete and other operations.

\section{Police force distribution inquiring}

The system can be distributed through the staff interface to view the various personnel under the distribution of information [5].

\section{Real-time staff distribution}

Provide real-time distribution of current (X minutes) online personnel (visible only on PC-side, under management authority).

Through its own institutions show the show all the staff under the real-time geographical distribution.

Different levels of patrolmen, different icons displayed on the map, are distinguished by different colors.

When the mouse is skipped to the location of the staff map, the system automatically emits the details of the person's information, including name, mobile phone number, affiliated organization, role, ID number and other information.

\section{Analysis of Force Distribution in Group}

The system provides different regional groups of anti-group power distribution of the thermal map; you can visually show the distribution of personnel in various regions.

System back-office administrator can learn from the thermal map of key areas of police deployment, can be in key areas, and in the event of an emergency, the police command and dispatch plays a key role. 


\section{Design of a key alarm module}

A key alarm module is in-depth consideration of the actual needs of the public security business designed to carry out the task of the patrol and the guards, when encountered an emergency threat to their lives, you can press this module to trigger the alarm function, with positioning function, Command center to the fastest response to the police, so as to protect the lives of field personnel.

A key alarm workflow is as follows:

1. Patrol officers found police intelligence;

2. Through the group defense through the police intelligence;

3. Group defense monitoring center received an alarm; notify the command and leadership, 110 command center received the alarm;

4. The command center issued a task, police around the $1 \mathrm{~km}$ to assist the support;

$5.1 \mathrm{~km}$ range of duty officers receives notice of the task push;

6. Investigators and assistants to report the results of the implementation of the task;

7. Command center monitoring task progress, report to the command and leadership in a timely manner;

8. The command center to close the task, and give the task implementation evaluation;

A key alarm module built a call to the phone program, long press a button alarm button, trigger the program, automatically call the police station where the duty phone, and access within $1 \mathrm{~km}$ within the patrol, the guards and security guards position, push the message give them.

The alarm person can send information such as location, text, voice and video to the police officer, and the police officer who receives the information can assist the support. This function gives police officers the opportunity to deal with dangerous situations, to ensure the safety of police officers and the efficient handling of emergencies.

\section{System performance optimization design}

\section{The design of the database}

Access to the database is one of the bottlenecks in system processing. For database access problems Traditional relational databases fail to meet the demands of speed and efficiency when dealing with very large and large concurrent web sites of the SNS type.

The system takes a variety of ways to effectively solve [6,7], including database connection pool applications, cache applications and data differentiation processing. Database connection pool solves the problem of system performance degradation caused by frequent database connection. Unique cache technology application makes the system greatly optimized in the case of large concurrent access. Data differentiation processing effectively separates historical data and current data so that the historical data Access to the query will not affect the efficiency of the current data processing, at the same time, effectively solve the years after the surge in data volume may bring about the performance of the system to protect the system to run the high reliability.

The system uses connection pooling to improve system performance, and for each connection application and return tracking, and strictly maintain the number of connections in the system performance allowable range; at full capacity operation will not show a significant performance degradation.

\section{Distributed File Server Design}

Distributed file server $[8,9]$

A file server is a device whose function is to provide a file to the server. It enhances the functionality of the memory and simplifies the management of network data. It improves the performance of the system, improves the availability of data, and reduces the complexity of management and reduces operating costs. 
The system uses FastDFS distributed file system to manage the file, including: file storage, file synchronization, file access (file upload, file download), etc., to solve the problem of mass storage and load balancing.

Distributed File Server Performance Test [10,11]

Taking into account the increase in the number of users and the data caused by the pressure to the server can not be estimated, so plans for FastDFS load performance test, the system configuration in the same circumstances, in a certain period of time, the server performance in high load performance performance, System environment for the correct analysis and evaluation.

The number of design threads is 100 , the concurrent number of concurrent requests of 2000, the test server stability and operational response time. Test tool: apache-jmeter-2.13 performance testing tools, test results shown in Figure 3.



Fig. 3. System concurrent pressure test

From the above figure we can see that the average response time of the image loading is $487 \mathrm{~ms}$, the curve is flat and the server loading process is smooth. The performance test curve in Figure 3 shows that the system is working properly under heavy load conditions, and the ability to handle data, stability and affordability meets the design requirements.

\section{Conclusions}

This article proposed based on the Java language group defense group management tower management system is highly intelligent, can help the public security bureau administrator to efficiently handle the police incident, patrol the first-line players can download the software to see scheduling, attendance, patrol And other information, the real fingertips on the police, and the design of a key alarm and intelligent scheduling and other practical help to help the police, greatly reducing the daily workload of the Public Security Bureau and the police station. System reliability, stability, and the application of the cache, distributed image server, database connection pool technology to ensure that even if the amount of user surge, the system's performance or remain within the allowable range, the system is now put into using.

\section{References}

[1] Lin Ziyu, Lai Yongxuan, Lin Chen. Cloud database research, Database technology, 2012,23(5):1149- 1153.

[2] Bruce Eckel. Java programming ideas, Translated by Chen Haopeng. Beijing: Machinery Industry Press, 2007.

[3] Tao Yizheng, Tang Dingyong, He Tiening, Li Qiuling, Zhou Nanhua, Lei Haihong. Research on Data Integration Framework of Heterogeneous Information System Based on Java and XML Technology, Computer Application Research, 2004,10(5):38- 41. 
[4] Dong Zhuoya. Communication map display based on Baidu map JavaScript API, Electronic design engineering, 2013,21(18):73- 75.

[5] Zhang Yu, Wang Yinghui, Zhang Xiangnan. Design and Implementation of MVC Framework Based on Spring, Computer engineering, 2010,36(4):59- 62.

[6] Wang Yinghui, Wang Yingjie, Wang Yanjun. Research and Implementation of Software Interface Architecture Based on MVC, Computer application research, 2004,21(8):188- 190.

[7] Chen Pingping, Tan Dingying, Liu Xiufeng. Research on Extensible Cloud Relational Database, Computer Engineering and Design, 2012,33(7):2691- 2693.

[8] Yang Yanlan, Jin Xiaoxue, Ye Hua.ASP.NET AJAX framework Research and Its Application in Web Development, Computer Applications and Software, 2011,28(6):195- 198.

[9] Rui Wanzhi, Jiang Hanhong, Hou Zhongyuan. Review and Prospect of Network Control System, Information and control,2012,41(1):83- 86.

[10]Zheng Meng, Zhang Qingling, Song Min, Jiang Chong. Stability Analysis of Short - time Multi packet Transmission Network Control System, Information and control, 2007,36(3):293- 297. 